\title{
Validación de la Escala de Esperanza para Emprender en la Mujer (EEEM)
}

\author{
Validation of the Hope for Female Entrepeneurship Scale
}

\author{
José María Vázquez-Espinosa ${ }^{1}$ Roberto Lagunes-Córdoba ${ }^{2}$ y Ana Delia López-Suárez ${ }^{3}$
}

\begin{abstract}
Resumen
La esperanza es un recorte de la realidad orientado a la consecución de metas, pues sienta las bases cognoscitivas (motivacionales y volitivas) para conseguirlas, por lo que tiene una relación cercana con el emprendimiento. No se encontraron escalas similares en mujeres, así que el propósito de este estudio fue validar una escala de esperanza para el emprendimiento femenino. El instrumento fue autoadministrado a una muestra de 331 mujeres mexicanas, entre 20 y 73 años. Se determinó la validez de constructo mediante un análisis factorial exploratorio. Se obtuvo una configuración de tres dimensiones con 14 reactivos: Agencia $(\omega=.88)$, Evaluación de rutas $(\omega=.79)$ y Planeación alterna $(\omega=.64)$, con índices de ajuste apropiados. Esta escala posee validez (contenido y constructo) y confiabilidad adecuadas, que permitirían utilizarla como una herramienta de evaluación, diagnóstico e investigación en las fases iniciales del emprendimiento.
\end{abstract}

Palabras clave: motivación, agencia, planeación, psicometría, validación

\begin{abstract}
Hope is a cognitive geared towards goal achievement, which establishes the cognitive basis (motivation and volition) for attaining goals, thus having a close relationship with entrepreneurship. There are no hope scales for female entrepreneurs, therefore, it is the aim of this research to validate a hope scale for female entrepreneurship. The questionnaire was self-administered to a sample of 331 Mexican women, aged between 20 and 73 years old. Construct validity was determined through exploratory factor analysis, obtaining a three-dimensional structure, considering 14 items: Agency $(\omega=.88)$, Pathways evaluation $(\omega=.79)$, and Alternative planning $(\omega=.64)$. It had adequate fit indexes. This scale possesses appropriate content and construct validity and reliability and may therefore be useful as a tool for assessment, diagnosis, and research in initial phases of entrepreneurship.
\end{abstract}

Keywords: motivation, agency, planning, psychometrics, validation

Nota: El estudiante de doctorado fue becado para estudios de este nivel por el Consejo Nacional de Ciencia y Tecnología.

\footnotetext{
${ }^{1}$ Maestro en Psicología del Trabajo y las Organizaciones. Estudiante de Doctorado en Psicología. Universidad Veracruzana. Instituto de Investigaciones Psicológicas. Av. Dr. Luis Castelazo Ayala s/n, C.P. 91190. Xalapa, Veracruz, México. Tel.: (+52) 2288127175. Correo: che_ma287@hotmail.com

${ }^{2}$ Doctor en Ciencias, con especialidad en neurobiología celular y molecular. Investigador académico de carrera, Titular C, Tiempo completo. Universidad Veracruzana. Instituto de Investigaciones Psicológicas. Av. Dr. Luis Castelazo Ayala s/n, C.P. 91190. Xalapa, Veracruz, México. Tel.: (+52) 2288135913. Correo: rlc.academico@yahoo.com.mx

3 Doctora en Psicología. Investigadora académico de carrera, Titular C, Tiempo completo. Universidad Veracruzana. Instituto de Investigaciones Psicológicas. Av. Dr. Luis Castelazo Ayala s/n, C.P. 91190. Xalapa, Veracruz, México. Tel.: (+52) 2288421700, ext. 13219. Correo: anadelia_lopez@yahoo.com (autora de correspondencia)

Revista Iberoamericana de Diagnóstico y Evaluación - e Avaliação Psicológica. RIDEP · No61 · Vol.4 · 19-32 2021

ISSN: 1135-3848 print /2183-6051online
} 


\section{Introducción}

\section{Emprendimiento}

El emprendimiento es ser pionero en algo, se generan ideas (Castiblanco, 2013) que permiten iniciar una empresa o proyecto; o bien, dar un valor agregado a un producto o servicio existente (Jaramillo, 2008), cuando se encuentra la oportunidad para hacerlo (McMullen \& Shepherd, 2006; Onuoha, 2007). Quienes emprenden van tras una meta deseada (McMullen \& Shepherd, 2006), en la que pueden incluir a otros, al crear puestos de trabajo (Markussen \& Roed, 2017).

\section{Esperanza}

Etimológicamente, la palabra esperanza proviene del término en latín sperare, derivado de spes, relacionado con una raíz indoeuropea traducida como expandirse. Dicho vocablo también se encuentra en el adjetivo latino prosperus, que se traduce como feliz. En griego, proviene del verbo sacar y extender (Diccionario de etimologías de Chile, 2019).

Si bien el constructo de esperanza se ha analizado desde la antigüedad, con diversas perspectivas, no existe un consenso en su definición científica. En psicología, esto se aprecia en que se entiende como un aspecto motivacional (motivación, deseo, anhelo; véase Bai, Kohli, \& Malik, 2017; McGuire-Snieckus, 2014; Snyder et al., 1996; Yang, Zhang, \& Kou, 2016), afectivo (emoción, confianza; véase Bruininks \& Malle, 2005; Scioli et al., 1997), cognoscitivo (creencias, expectativa, fantasía positiva, optimismo, capacidad para planear; véase Gallagher, 2018; Oettingen, 1997), como un rasgo de personalidad, un estado (recurso psicológico susceptible de cambiarse) que es parte de los cuatro componentes del capital psicológico (Luthans \& Youssef, 2004; Mahfud, Triyono, Sudira, \& Mulyani, 2019), una actitud (véase Snyder, Lopez, Shorey, Rand, \& Feldman, 2003) o una capacidad (habilidad, capacidad percibida; véase Callina, Snow, \& Murray, 2018).

En general, se identifican dos grandes líneas psicológicas para definir a la esperanza. Por un lado, la ubican como una emoción y por otro, como una cognición. Sin embargo, hoy en día se reconoce que estas categorías se traslapan y que el constructo tiene ambas cualidades (Lopez, Snyder, \& Teramoto 2004).

En la primera versión de la teoría de la esperanza de Snyder, Irving y Anderson (1991), ésta se conceptualizó como un enfoque del pensamiento que deriva de un sentido de éxito, originado a partir de una relación de reciprocidad entre dos elementos: la agencia (determinación dirigida hacia la meta) y las rutas (planeación de diferentes maneras de conseguir metas). La esperanza es un pensamiento dirigido hacia la meta, en el cual la persona identifica diversos caminos para alcanzarla (pensamiento de rutas) y está motivada para implementar las rutas elegidas (pensamiento de agencia; Lopez et al., 2004).

De manera similar, para Callina et al. (2018), la esperanza es un estado motivacional positivo de corte cognitivo, caracterizado por una fuerte agencia y por un pensamiento de planeación alterna, que brinda tanto la determinación para el logro, como las herramientas cognitivas para alcanzar exitosamente los propósitos que se tenían en mente.

De acuerdo con estas definiciones, los elementos fundamentales de la esperanza son la agencia y las rutas. La agencia es un elemento motivacional (Snyder, Rand, \& Sigmon, 2002) que da a una persona la determinación y la certeza para iniciar el esfuerzo que la llevará a conseguir la meta deseada, de mantenerse en ese intento (Valle, Huebner, \& Suldo, 2006), y de persistir ante un obstáculo (Dixson, Worrell, \& Mello, 2017).

Las rutas consisten en evaluar si la meta elegida es alcanzable y en desarrollar múltiples formas de conseguirla (Hutz, Midgett, \& Cerentini Pacico, 2014; Snyder, Irving et al., 1991; Snyder et al., 1996), ubicando acciones que permitan sobreponerse a los obstáculos mediante la generación de planes alternativos.

Scioli et al. (1997) reconocen que la esperanza motiva la acción y afecta tanto a los pensamientos, como al comportamiento. Ésta contiene muchos de los pasos seguidos para lograr una meta, pero no se ve como proceso, sino como un constructo que permite a la persona encuadrar al logro como algo realizable: incluye la motivación para ir tras una meta, determinar su viabilidad y analizar la propia capacidad para obtener el resultado; ponerla bajo control, al 
planear y anticipar los posibles obstáculos y, por ende, generar soluciones.

Por su parte, Oettingen y Gollwitzer (2002) indican que si las creencias de esperanza asociadas con la agencia son fuertes, debería surgir un compromiso que inicie las acciones requeridas para alcanzar la meta deseada. $\mathrm{Al}$ ser así, la esperanza puede considerarse la fase inicial del proceso para conseguir algo; en otras palabras, ésta se convierte en el antecedente de la acción y del logro. La empresa o negocio ya establecido es justamente el resultado del emprendimiento, es la culminación del proceso de consecución.

La definición de esperanza para este trabajo derivó de un análisis de la literatura existente y de las diferentes propuestas teóricas. Nos inclinamos por una visión de la esperanza desde lo cognoscitivo, porque constituye una manera de interpretar la información existente y de pensar acerca del futuro con un encuadre particular; que motiva y da certeza de lo que la persona es capaz, lo que da paso a las acciones de premeditación y planeación que permitirán tener un resultado exitoso.

Dado lo anterior, se desprende el término esperanza para emprender. Éste se definió como un encuadre cognoscitivo para interpretar la información existente y pensar acerca de un futuro prometedor, de tal manera que la persona esté motivada y tenga certeza para iniciar un negocio, teniendo varios planes en mente y preparándose anticipadamente para sobreponerse a los obstáculos. En un primer momento, consideramos que constaba de dos dimensiones: agencia y rutas de acción para el logro, por lo que así se hizo el plan de prueba.

La agencia son las creencias personales acerca de la capacidad para lograr determinado resultado y la motivación que le permite orientarse a largo plazo para lograr sus metas en un futuro prometedor. Las rutas de acción para el logro son procesos cognoscitivos destinados a: evaluar si la meta de emprender un negocio es alcanzable, y a desarrollar múltiples formas de conseguirla (Hutz et al., 2014; Snyder, Irving et al., 1991; Snyder et al., 1996), con acciones y planes alternos.

\section{La mujer emprendedora en México}

Una persona emprendedora identifica una oportunidad de negocio y es capaz de asumir el riesgo calculado para crear y mantener una nueva empresa innovadora o bien, partir de conceptos ya existentes (Lambing \& Kuehl, 1998). Para tomar decisiones, considera tanto sus características personales, como el comportamiento y las características contextuales del mercado laboral (Osowska, Kapasi, \& Jackman, 2016). Asimismo, es propietaria de la mayor parte de la empresa y realiza los trámites administrativos para su apertura y desarrollo (Portillo Navarro \& Millán Jiménez, 2016).

Una persona que trabaja como multinivel es representante de marca en una empresa organizada en red jerárquica de vendedores, relativamente independientes, cuyos ingresos son proporcionales al trabajo propio y al de quienes están en niveles inferiores dentro de su estructura de colaboradores (Alvaro Fariñas, 2015).

Se requiere acentuar las diferencias entre emprendores y multinivel con la finalidad de sentar las bases de este estudio, al emplear la comparación entre los factores resultantes, como validez predictiva. Emprendedores y multinivel son diferentes, porque los primeros innovan y asumen mayor cantidad de inversión, de responsabilidad y de riesgo: invierten en infraestructura, materiales, salario de empleados o pago de los servicios que requieran, entre otros recursos que, generalmente, provienen del patrimonio personal.

Los multinivel requieren de poca inversión económica, por lo que el riesgo es menor; no podrían considerarse pioneros, ya que inician su negocio insertos en una estructura que los acoge, en la cual generalmente reciben capacitación. Quizá pudieran ser pioneros al desarrollar alguna estrategia para la expansión de su red. De igual manera, pueden o no generar puestos de trabajo.

Actualmente, en México, el emprendimiento femenino (16.84\%) aventaja grandemente al masculino (0.23\%; Rubio \& Esteban, 2016), pues de las casi 1,200,000 de pequeñas y medianas empresas existentes en el país, alrededor de 340,000 se encuentran en manos de mujeres (Etchegoyen, 2019), que generalmente son jefas de familia o que buscan tener cierta libertad económica (Castiblanco, 2016). A pesar de estas estadísticas, el estudio del emprendimiento femenino y la importancia de su situación particular como jefas de familia es escaso y se 
Tabla 1. Instrumentos para la medición psicológica de la esperanza

\begin{tabular}{|c|c|c|c|}
\hline Autores & Ámbito de medición & Dimensiones/Reactivos & $\alpha$ \\
\hline Stotland (1969) & $\begin{array}{l}\text { Expectativa superior a cero del logro } \\
\text { de una meta }\end{array}$ & & .82 \\
\hline Gottschalk, (1974) & Hope Scale & 7 dimensiones & .82 \\
\hline Erickson, Post y Paige (1975) & Hope Scale & 20 reactivos & .79 \\
\hline Stoner y Keampfer (1985) & Stoner Hope Scale & 10 adjetivos & .93 \\
\hline Obayuwana et al. (1982) & The Hope Index Scale & 50 reactivos & .88 \\
\hline Nowotny (1989) & Nowotny Hope Scale & $\begin{array}{l}47 \text { reactivos } 1 \text { ) orientación al futuro, 2) } \\
\text { participación, 3) motivación interna, 4) Lo } \\
\text { que es posible, 5) involucrar a otros o a un } \\
\text { ser superior, 6) resultados significativos de } \\
\text { los individuos }\end{array}$ & .90 \\
\hline & & $\begin{array}{l}40 \text { reactivos } 1 \text { ) Relaciones con los demás, } 2 \text { ) } \\
\text { sentido de competencia personal, } 3 \text { ) }\end{array}$ & \\
\hline Miller y Powers (1988) & The Miller Hope Scale & $\begin{array}{l}\text { capacidad de afrontamiento, 4) bienestar } \\
\text { psicológico, 5) propósito y 6) significado en } \\
\text { la vida }\end{array}$ & .93 \\
\hline Hert (1991) & The Hert Hope Scale & 30 reactivos & $.89-.91$ \\
\hline Snyder, Harris et al. (1991) & Adult Dispositional Hope Scale & $\begin{array}{l}\text { Agencia y rutas } \\
8 \text { reactivos }\end{array}$ & $.70-.80$ \\
\hline Vargas Núñez (1995) & Escala de Esperanza & 53 reactivos en escala tipo Likert & \\
\hline $\begin{array}{l}\text { Babyak, Snyder y Yoshinobu } \\
\text { (1993) }\end{array}$ & Escala de Esperanza & Agencia y rutas & $.96-.99$ \\
\hline Snyder et al. (1997) & Children's Hope Scale & $\begin{array}{l}\text { Agencia y rutas } \\
8 \text { reactivos }\end{array}$ & .70 \\
\hline $\begin{array}{l}\text { Martínez Uribe, Cassaretto } \\
\text { Bardales y Hert (2012) }\end{array}$ & $\begin{array}{l}\text { Escala de Esperanza de Hert } \\
\text { (validación en español) }\end{array}$ & 30 reactivos & .89 \\
\hline Villacieros et al. (2017) & $\begin{array}{l}\text { Escala de Esperanza en Enfermedad } \\
\text { Terminal (EEET) }\end{array}$ & $\begin{array}{l}\text { Relaciones de trascendencia y relaciones } \\
\text { personales }\end{array}$ & $.76-.87$ \\
\hline
\end{tabular}

Fuente: Elaboración propia, a partir de las referencias consultadas y que aparecen en el apartado correspondiente.

carece de instrumentos con contenidos dirigidos específicamente hacia su situación y circunstancias particulares.

La esperanza contiene aspectos que se han atribuido a personas emprendedoras, tales como: establecer metas, afrontar riesgos o ser creativos (Bruininks \& Malle, 2005; Hutz et al., 2014; Snyder, 1995). Paes (2002) indica que el trabajador de una empresa multinivel también posee los rasgos de autoestima, autoconfianza y determinación, igual a los señalados en la literatura de emprendedores (Veáse: Bullough \& Renko, 2013); nótese que la autoconfianza y la determinación son subdimensiones de la agencia, descrita en el constructo de esperanza.

\section{Esperanza para emprender}

Hasta el momento, las escalas construidas para medir la esperanza se basan en definiciones que la consideran una característica general, que indica un posicionamiento o evaluación hacia la vida $\mathrm{y}$, se han centrado en la parte cognitivoemotiva, aplicadas a un contexto de salud (i.e. salud mental, cuidados terminales, enfermedades crónicas, entre otros). Psicométricamente, se han identificado las dimensiones: cognitivo temporal, afectivo conductual y afiliativo contextual (Hert, 1991); relación con seres queridos (Nowotny, 1989), con Dios o la trascendencia y con uno mismo (Villacieros, Bermejo, \& Hassoun, 2017) o agencia y rutas (Snyder, Harris et al., 1991); la confiabilidad general ha variado entre .72 y .94 . Los instrumentos encontrados se describen en la Tabla 1.

La esperanza no se ha abordado en su vinculación con el logro de metas, a pesar de que conceptualmente se asocia con este aspecto, según Lopez et al. (2004). Estos autores refieren varios problemas en la medición y evaluación de la esperanza, el primero consiste en la complejidad para definir el constructo y que ahonda en confusión y ambigüedad. El segundo se refiere a las técnicas para medirla: observación directa o con escalas de autorreporte. Asimismo, refieren la preocupación de tener una medición certera del atributo de la esperanza en las personas. Por eso, como indican Oliveira, Merino, Privado y Almeida (2017) es importante invertir en la construcción y validación de instrumentos capaces de evaluar las dimensiones psicológicas seleccionadas. 
Tabla 2. Datos demográficos de la muestra

\begin{tabular}{|c|c|c|c|c|c|c|}
\hline & \multicolumn{2}{|c|}{$\begin{array}{c}\text { Todas } \\
\mathrm{N}=331\end{array}$} & \multicolumn{2}{|c|}{$\begin{array}{c}\text { Emprendedoras } \\
n=165\end{array}$} & \multicolumn{2}{|c|}{$\begin{array}{c}\text { Multinivel } \\
n=166\end{array}$} \\
\hline & $f$ & $\%$ & $f$ & $\%$ & $f$ & $\%$ \\
\hline \multicolumn{7}{|l|}{ Estado civil } \\
\hline Soltera & 104 & 31.4 & 52 & 31.5 & 52 & 31.3 \\
\hline Casada & 112 & 33.8 & 55 & 33.3 & 57 & 34.3 \\
\hline Separada & 47 & 14.2 & 22 & 13.3 & 25 & 15.1 \\
\hline Viuda & 16 & 4.8 & 9 & 5.5 & 7 & 4.2 \\
\hline Unión libre & 52 & 15.7 & 27 & 16.4 & 25 & 15.1 \\
\hline \multicolumn{7}{|l|}{ Escolaridad } \\
\hline Ninguna & 6 & 1.8 & 3 & 1.8 & 3 & 1.8 \\
\hline Primaria & 48 & 14.5 & 24 & 14.5 & 24 & 14.5 \\
\hline Secundaria & 71 & 21.5 & 30 & 18.2 & 41 & 24.7 \\
\hline Bachillerato & 89 & 26.9 & 41 & 24.8 & 48 & 28.9 \\
\hline Carrera técnica & 50 & 15.1 & 25 & 15.2 & 25 & 15.1 \\
\hline Licenciatura & 57 & 17.2 & 32 & 19.4 & 25 & 15.1 \\
\hline \multirow[t]{2}{*}{ Posgrado } & 10 & 3.0 & 10 & 6.1 & 0 & 0 \\
\hline & $M$ & $D E$ & $M$ & $D E$ & $M$ & $D E$ \\
\hline $\begin{array}{l}\text { Tiempo con el negocio } \\
\text { establecido }\end{array}$ & 7.80 & 5.34 & 8.30 & 6.19 & 7.30 & 4.30 \\
\hline
\end{tabular}

Nota. El nivel bachillerato equivale a un nivel medio superior, previo al ingreso a la universidad.

Después de una búsqueda en la literatura científica sobre esperanza, no se ubicó una escala que estuviera orientada al emprendimiento. Los instrumentos sobre emprendimiento se enfocan en la intención emprendedora como el Entrepreneurial Intention Questionnaire (EIQ) de Liñán y Chen (2009), basado en el modelo de potencial emprendedor de Krueger, Reilly y Carsrud (2000) que mide dimensiones como actitudes personales, normas subjetivas, control percibido de comportamiento y espíritu empresarial (Moriano, Gorgievski, Laguna \& Stephan, 2012) o a la orientación emprendedora (Covin \& Wales, 2011): Escala de Miller Covin (1989) y Escala de Hughes y Morgan (2007).

El estudio de Moses, Olokundun, Akinbode, Agboola e Inelo (2016) vincula al emprendimiento con el optimismo y con la pasión, pero no se encontraron investigaciones que relacionen a la esperanza con el emprendimiento.

Dada la situación particular de las mujeres emprendedoras y la falta de instrumentos válidos y confiables para estudiar la esperanza para emprender, la investigación en esta temática requiere el diseño de una escala de esperanza en el inicio y el desarrollo de un negocio ya establecido. El propósito del estudio fue construir y validar una escala de esperanza para el emprendimiento femenino.

\section{Método}

El estudio fue retrospectivo e instrumental. Retrospectivo porque se indagó acerca del inicio y desarrollo de un negocio establecido, e instrumental porque se refiere al desarrollo de nuevos procedimientos, aparatos, instrumentos o pruebas, para determinar sus propiedades psicométricas (Montero \& León, 2007).

\section{Participantes}

Se abordó a 336 mujeres, provenientes de la capital de un estado del sureste de la República Mexicana y de sus poblaciones más cercanas; 165 fueron definidas como emprendedoras, 166 como multinivel, y se eliminaron 5 por no cumplir los criterios de inclusión.

Se ubicó a las mujeres como emprendedoras cuando tenían un negocio propio y que hubieran realizado todos los trámites administrativos para su apertura; y multinivel cuando trabajaban en una empresa de este tipo, según se explicó anteriormente. Los criterios de inclusión fueron: mujeres que hayan sido propietarias únicas del negocio, y que el negocio tuviera, al menos, tres años y medio funcionando ininterrumpidamente. Este criterio se sustenta en la clasificación del Global Entrepreneurship Monitor (GEM, 2017), que considera al emprendimiento establecido el que va de tres años y medio a cinco años, porque el negocio tiene el tiempo suficiente en el mercado como para tener clientes y resistir, por lo 
menos, los primeros obstáculos, lo cual implica que la meta de establecer una empresa se hubiera logrado.

El muestreo fue no aleatorio, tipo bola de nieve (Clark-Carter, 2004; Hair, Bush, \& Ortinau, 2010), que se utilizó porque la muestra de emprendedoras fue difícil de encontrar; pues aunque se agrupaban para capacitarse o para apoyarse, el lugar de reunión no era del dominio público. Algunas no pertenecían a ninguna agrupación.

La edad de las participantes osciló entre 20 y 73 años $(M=39.26, D E=10.91)$. El estado civil tuvo como moda la opción casadas, tanto para las emprendedoras $(\mathrm{n}=55)$, como para las multinivel $(n=57)$. El resto de las características sociodemográficas de la muestra se aprecian en la Tabla 2.

\section{Instrumento}

La Escala de Esperanza para emprender en la Mujer (EEEM) se conformó por 29 reactivos, que debían responderse a partir de la frase: "Al inicio y desarrollo de mi negocio, yo...". Éstos tuvieron un formato de respuesta en escala tipo Likert, con cinco opciones de respuesta, que iban desde "nunca" (1) hasta "siempre" (5). Adicionalmente, se aplicó un cuestionario que solicitaba información acerca de datos sociodemográficos (edad, escolaridad, estado civil) y relacionados con su negocio (tiempo de tener el negocio, tipo de negocio, si contaba con empleados). Esta última sección sirvió para confirmar que la participante cumplía con los criterios de inclusión.

\section{Procedimiento de construcción}

\section{a) Jueceo del instrumento}

Inicialmente se diseñaron 33 reactivos que se presentaron a jueces expertos en construcción de instrumentos para determinar su validez de facie y de contenido. Lo anterior se llevó a cabo en tres etapas. En la primera etapa intervinieron dos psicólogos, en la segunda tres psicólogos sociales, y en la tercera un psicómetra. La primera etapa del jueceo permitió resolver problemas de definición de las dimensiones, ya que al intentar construir los reactivos se encontró poca claridad conceptual en los modelos teóricos, y eso provocó que las dimensiones se traslaparan. Una vez corregido este defecto, se llevó a cabo el segundo jueceo.
En esta segunda etapa, se pidió a tres jueces que evaluaran la validez de contenido del instrumento, la cual describe la forma en que los reactivos diseñados responden a la dimensión que se intenta medir (Ramos et. al, 2020). Para ello, se pidió que los jueces indicaran si los reactivos: (a) eran congruentes teóricamente con el constructo, y (b) correspondían a la dimensión en la que estaban propuestos. Los jueces debían recomendar si se conservaba el reactivo, y si se quitaba o si se cambiaba su redacción (para eliminar sesgos de respuesta); además se solicitaron observaciones adicionales sobre la redacción, para que fuera adecuada a las características de la población objetivo.

En esta etapa, la confiabilidad interjueces se llevó a cabo de manera cualitativa, se discutió cualquier diferencia hallada entre los jueces. En cada reactivo, cuando hubo un $100 \%$ o un $66 \%$ de acuerdo en la observación, se atendía y cuando tuvo un 33\% se discutía. Una vez concluido este proceso, se eliminaron cuatro reactivos y se hicieron los cambios necesarios a la redacción, pero no se modificaron las dimensiones propuestas al instrumento.

En la tercera etapa se verificó la conformación final del instrumento, ya con 29 reactivos.

\section{b) Recolección de datos}

La muestra se contactó en diferentes espacios: mercados ambulantes, negocios establecidos formalmente, agrupaciones de emprendedoras o multinivel, así como grupos de mujeres en redes sociales dedicados a negocios. La invitación fue telefónica en 25 casos, y por correo electrónico en 11. Durante el primer contacto, se les explicó el propósito del estudio y en qué consistiría su participación. En caso de aceptar, se procedía a fijar una cita para aplicar el instrumento, la cual duró aproximadamente 15 minutos, fue presencial y en su mayoría fue individual; pues hubo tres sesiones de aplicación grupales con 11 personas como máximo.

Se siguió un formato de autoaplicación; el aplicador únicamente leyó las instrucciones en voz alta y aclaró las dudas. Las participantes leyeron y firmaron un consentimiento informado, en donde se indicaba la finalidad del estudio; que su participación sería voluntaria y sin retribución económica; que los datos proporcionados serían 
anónimos, confidenciales, empleados con fines de investigación y que podían abandonar la investigación en el momento en que así lo dispusieran. También se indicó cuál sería el procedimiento para proteger sus respuestas. Como retribución a su colaboración, se ofreció un curso sobre habilidades de gestión para formalizar el empresa.

Se invitó a 25 alumnos de la licenciatura en trabajo social, 23 mujeres y 2 hombres, para fungir como aplicadores, quienes se capacitaron para estandarizar el proceso. Su participación no tuvo ningún tipo de retribución económica o académica. Previo al trabajo de campo, los estudiantes firmaron un acuerdo de confidencialidad. El protocolo de investigación fue aprobado por el comité de ética del instituto de investigación al que pertenecen los investigadores, con el folio 201804.

\section{c) Análisis psicométrico}

El análisis de los datos se llevó a cabo con el Statistical Package for the Social Sciences (SPSS), versión 22. A continuación, se describe el procedimiento.

En primer lugar se verificó que no hubiera ningún dato faltante, se procedió a calcular el cociente de asimetría, con el cual, según la recomendación de Ferrando y Anguiano-Carrasco (2010), se deben eliminar los reactivos que tengan un cociente de asimetría mayor que 3, o menor que -3 . El ajuste de los reactivos a una distribución normal se determinó mediante la prueba de Kolmogorov-Smirnov.

También se comprobó que todas las opciones de respuesta propuestas hubieran sido elegidas, para decidir si el número de opciones posibles era adecuado.

Posteriormente, se realizó el análisis de poder discriminativo por medio de la técnica de grupos contrastantes. Para obtenerlo, se dividió a la muestra en dos grupos, conformados a partir del primer y el cuarto cuartil de los puntajes de cada reactivo. Luego, se compararon las puntuaciones de ambos cuartiles por medio de la prueba t de Student para muestras independientes (Nunnally, 1991).

La validez de constructo se determinó mediante el análisis factorial exploratorio (AFE). Cuando las puntuaciones de los reactivos no se ajustan a la distribución normal, se lleva a cabo el
AFE con el método de extracción de mínimos cuadrados no ponderados (Flora, LaBrish, \& Chalmers, 2012; Ximénez \& García, 2005) con rotación oblicua (normalización Kaiser), para reflejar de forma más precisa la estructura de la escala, en función de la interacción de los factores propuestos, pues debido a la naturaleza del constructo se asume que están correlacionados (Beavers, Iwata, \& Lerman, 2013; Williams, Onsman, \& Brown, 2010).

Tras el primer AFE, se exploró la matriz de correlación entre los factores, para identificar el porcentaje de correlaciones superiores a .32; y decidir entre un método de rotación oblicua o uno ortogonal, de acuerdo con la propuesta de Tabachnick y Fidelli (2001).

Se hicieron tantas rondas de AFE como fue necesario, hasta obtener una estructura factorial estable estadísticamente y congruente conceptualmente. Para conseguirlo, se conservaron los reactivos que tenían carga factorial superior a $.40 \mathrm{y}$ una comunalidad igual o superior a .30 ; se eliminaron los reactivos que no se ubicaron en ningún factor y aquéllos que conceptualmente no pertenecían al factor en el que se encontraban.

La adecuación de los datos al modelo factorial se determinó mediante: la prueba de esfericidad de Bartlett, la determinante de la matriz de correlaciones y la prueba de adecuación muestral Kaiser-Meyer-Olkin (Beavers et al., 2013). Dada la falta de normalidad de los datos obtenidos, la confiabilidad de los factores de la escala se obtuvo mediante el coeficiente omega de McDonald (McDonald, 1999).

Para determinar la validez predictiva de la escala, se analizaron las puntuaciones de las mujeres emprendedoras y las multinivel para la escala total y sus dimensiones, con una prueba de diferencias para muestras independientes.

\section{Resultados}

Se llevó a cabo una sola aplicación, en la cual se duplicó el criterio mínimo de 5 personas por reactivo (Nunnally, 1991), al tener 29 reactivos y 331 participantes.

Los reactivos tuvieron puntuaciones superiores a la media teórica, pues las medias fluctuaron desde 3.57 hasta 4.53 , con desviaciones estándar de .81 a 1.35; todos tuvieron una distribución no normal, 
Tabla 3. Caracterización de los reactivos del instrumento y toma de decisión final

\begin{tabular}{|c|c|c|c|c|c|c|c|c|c|c|}
\hline & Reactivo & Dimensión & $M$ & $D S$ & $\begin{array}{l}\text { Cociente de } \\
\text { asimetría }\end{array}$ & Kurtosis & $\begin{array}{c}\mathrm{Z} \text { de } \\
\text { Kolmogorov- } \\
\text { Smirnov } \\
\end{array}$ & $R h o^{+}$ & Discriminación & Decisión \\
\hline 1 & $\begin{array}{l}\text { Pensaba en los resultados que } \\
\text { tendría al emprender mi negocio }\end{array}$ & Rutas & 3.82 & 1.35 & -0.83 & -0.57 & $.27^{*}$ & $.40^{*}$ & $\begin{array}{c}t(141.05)=-10.42 \\
p<.001\end{array}$ & $\begin{array}{l}\text { Eliminado } \\
\text { AFE }\end{array}$ \\
\hline 2 & $\begin{array}{l}\text { Sabía que me sería posible tener mi } \\
\text { negocio }\end{array}$ & Agencia & 3.98 & 1.21 & -0.94 & -0.20 & $.28^{*}$ & $.45^{*}$ & $\begin{array}{c}t(139.23)=-10.67 \\
p<.001\end{array}$ & $\begin{array}{l}\text { Eliminado } \\
\text { AFE }\end{array}$ \\
\hline 3 & $\begin{array}{l}\text { Tenía en mente varias opciones } \\
\text { para lograr emprender mi negocio }\end{array}$ & Rutas & 3.83 & 1.28 & -0.86 & -0.38 & $.24^{*}$ & $.45^{*}$ & $\begin{array}{c}t(151.19)=-10.12 \\
p<.001\end{array}$ & $\begin{array}{l}\text { Eliminado } \\
\text { AFE }\end{array}$ \\
\hline 4 & $\begin{array}{l}\text { Pensé que tendría un futuro mejor } \\
\text { al emprender mi negocio }\end{array}$ & Agencia & 4.18 & 1.06 & -1.20 & 0.63 & $.30^{*}$ & $.47 *$ & $\begin{array}{c}t(107.22)=-10.66 \\
p<.001\end{array}$ & $\begin{array}{l}\text { Eliminado } \\
\text { AFE }\end{array}$ \\
\hline 5 & $\begin{array}{l}\text { Resolví los problemas que se iban } \\
\text { presentando }\end{array}$ & Rutas & 4.23 & 0.94 & -1.07 & 0.34 & $.30^{*}$ & $.56^{*}$ & $\begin{array}{c}t(114.79)=-12.03 \\
p<.001\end{array}$ & $\begin{array}{l}\text { Eliminado } \\
\text { AFE }\end{array}$ \\
\hline 6 & $\begin{array}{l}\text { Creí en mí para llegar a la meta de } \\
\text { emprender mi negocio }\end{array}$ & Agencia & 4.40 & 0.89 & -1.46 & 1.45 & $.36^{*}$ & $.56^{*}$ & $\begin{array}{c}t(97.63)=-10.81 \\
p<.001\end{array}$ & Agencia \\
\hline 7 & $\begin{array}{l}\text { Desarrollé acciones efectivas para } \\
\text { resolver aspectos fuera de mi } \\
\text { control }\end{array}$ & Rutas & 3.79 & 1.13 & -0.79 & 0.03 & $.22^{*}$ & $.40 *$ & $\begin{array}{c}t(168.97)=-7.08 \\
p<.001\end{array}$ & $\begin{array}{l}\text { Eliminado } \\
\text { AFE }\end{array}$ \\
\hline 8 & $\begin{array}{l}\text { Disfruté el proceso de emprender } \\
\text { mi negocio }\end{array}$ & Agencia & 4.23 & 0.98 & -1.14 & 0.64 & $.31^{*}$ & $.59 *$ & $\begin{array}{c}t(107.31)=-12.12 \\
p<.001\end{array}$ & $\begin{array}{l}\text { Eliminado } \\
\text { AFE }\end{array}$ \\
\hline 9 & $\begin{array}{l}\text { Tenía una idea clara sobre aquello } \\
\text { que quería lograr }\end{array}$ & Agencia & 4.28 & 0.97 & -1.45 & 1.83 & $.31^{*}$ & $.54 *$ & $\begin{array}{c}t(104.67)=-10.74 \\
p<.001\end{array}$ & $\begin{array}{l}\text { Eliminado } \\
\text { AFE }\end{array}$ \\
\hline 10 & $\begin{array}{l}\text { Consideré los obstáculos a los que } \\
\text { me enfrentaría }\end{array}$ & Rutas & 3.71 & 1.27 & -0.70 & -0.56 & $.21^{*}$ & $.63^{*}$ & $\begin{array}{c}t(162.43)=-12.99 \\
p<.001\end{array}$ & $\begin{array}{l}\text { Evaluación de } \\
\text { rutas }\end{array}$ \\
\hline 11 & $\begin{array}{l}\text { Analicé que tan posible era lograr } \\
\text { lo que tenía en mente }\end{array}$ & Rutas & 3.90 & 1.15 & -0.89 & -0.02 & $.22^{*}$ & $.71^{*}$ & $\begin{array}{c}\mathrm{t}(140.91)=-14.65 \\
p<.001\end{array}$ & $\begin{array}{l}\text { Evaluación de } \\
\text { rutas }\end{array}$ \\
\hline 12 & $\begin{array}{l}\text { Cambié mi planeación para lograr } \\
\text { el emprendimiento, cuando fue } \\
\text { necesario }\end{array}$ & Rutas & 3.57 & 1.32 & -0.66 & -0.65 & $.22^{*}$ & $.53 *$ & $\begin{array}{c}\mathrm{t}(175.00)=-7.47 \\
p<.001\end{array}$ & $\begin{array}{l}\text { Planeación } \\
\text { alterna }\end{array}$ \\
\hline 13 & $\begin{array}{l}\text { Fui persistente para lograr mi } \\
\text { objetivo de emprender mi negocio }\end{array}$ & Agencia & 4.37 & 0.88 & -1.42 & 1.75 & $.35^{*}$ & $.59 *$ & $\begin{array}{c}\mathrm{t}(106.31)=-9.93 \\
p<.001\end{array}$ & Agencia \\
\hline 14 & $\begin{array}{l}\text { Dividí en pequeños pasos el } \\
\text { emprendimiento de mi negocio } \\
\text { para lograrlo }\end{array}$ & Rutas & 3.80 & 1.20 & -0.76 & -0.37 & $.21^{*}$ & $.59 *$ & $\begin{array}{c}\mathrm{t}(167.16)=-9.44 \\
p<.001\end{array}$ & $\begin{array}{l}\text { Planeación } \\
\text { alterna }\end{array}$ \\
\hline 15 & $\begin{array}{l}\text { Creí en mi capacidad } \\
\text { emprendedora }\end{array}$ & Agencia & 4.40 & 0.93 & -1.69 & 2.51 & $.36^{*}$ & $.57 *$ & $\begin{array}{c}\mathrm{t}(116.43)=-9.15 \\
p<.001\end{array}$ & Agencia \\
\hline 16 & $\begin{array}{l}\text { Tomé varios caminos para } \\
\text { conseguir lo que tenía en mente }\end{array}$ & Rutas & 3.72 & 1.31 & -0.79 & -0.51 & $.23 *$ & $.56^{*}$ & $\begin{array}{c}\mathrm{t}(175.00)=-5.54 \\
p<.001\end{array}$ & $\begin{array}{l}\text { Planeación } \\
\text { alterna }\end{array}$ \\
\hline 17 & $\begin{array}{l}\text { Me creí capaz de emprender mi } \\
\text { negocio }\end{array}$ & Agencia & 4.53 & 0.81 & -1.90 & 3.40 & $.40^{*}$ & $.60^{*}$ & $\begin{array}{c}\mathrm{t}(93.30)=-10.27 \\
p<.001\end{array}$ & Agencia \\
\hline 18 & $\begin{array}{l}\text { Creí era capaz de realizar las } \\
\text { acciones necesarias para emprender } \\
\text { mi negocio }\end{array}$ & Agencia & 4.26 & 0.97 & -1.33 & 1.29 & $.31 *$ & $.50^{*}$ & $\begin{array}{c}\mathrm{t}(101.91)=-11.56 \\
p<.001\end{array}$ & $\begin{array}{l}\text { Eliminado } \\
\text { AFE }\end{array}$ \\
\hline 19 & $\begin{array}{l}\text { Orienté mis acciones hacia lograr } \\
\text { emprender mi negocio }\end{array}$ & Agencia & 4.12 & 0.97 & -1.05 & 0.79 & $.25^{*}$ & $.47^{*}$ & $\begin{array}{c}\mathrm{t}(169.04)=-9.13 \\
p<.001\end{array}$ & $\begin{array}{l}\text { Eliminado } \\
\text { AFE }\end{array}$ \\
\hline 20 & $\begin{array}{l}\text { Tomé en cuenta los imprevistos } \\
\text { que pudieran surgir }\end{array}$ & Rutas & 3.75 & 1.21 & -0.72 & -0.39 & $.20^{*}$ & $.61^{*}$ & $\begin{array}{c}\mathrm{t}(127.25)=-14.49 \\
p<.001\end{array}$ & $\begin{array}{l}\text { Evaluación de } \\
\text { rutas }\end{array}$ \\
\hline 21 & $\begin{array}{l}\text { Me sentí motivada para hacer lo } \\
\text { necesario para emprender mi } \\
\text { negocio }\end{array}$ & Agencia & 4.38 & 0.87 & -1.43 & 1.57 & $.34^{*}$ & $.60^{*}$ & $\begin{array}{c}\mathrm{t}(129.18)=-12.26 \\
p<.001\end{array}$ & $\begin{array}{l}\text { Eliminado } \\
\text { AFE }\end{array}$ \\
\hline 22 & $\begin{array}{l}\text { Tenía la firme idea de establecer mi } \\
\text { negocio }\end{array}$ & Agencia & 4.31 & 1.03 & -1.59 & 1.95 & $.35^{*}$ & $.63^{*}$ & $\begin{array}{c}\mathrm{t}(97.81)=-11.41 \\
p<.001\end{array}$ & Agencia \\
\hline 23 & $\begin{array}{l}\text { Tuve interés en emprender mi } \\
\text { negocio }\end{array}$ & Agencia & 4.46 & 0.89 & -1.86 & 3.18 & $.38^{*}$ & $.57^{*}$ & $\begin{array}{c}\mathrm{t}(92.42)=-10.03 \\
p<.001\end{array}$ & Agencia \\
\hline 24 & $\begin{array}{l}\text { Me comprometí para cumplir la } \\
\text { meta de emprender mi negocio }\end{array}$ & Agencia & 4.46 & 0.85 & -1.65 & 2.41 & $.38^{*}$ & $.61^{*}$ & $\begin{array}{c}\mathrm{t}(97.47)=-11.44 \\
p<.001\end{array}$ & Agencia \\
\hline 25 & $\begin{array}{l}\text { Imaginé en mi mente lo que podría } \\
\text { hacer para emprender mi negocio }\end{array}$ & Rutas & 4.20 & 0.96 & -1.30 & 1.51 & $.27 *$ & $.59 *$ & $\begin{array}{c}\mathrm{t}(105.71)=-13.24 \\
p<.001\end{array}$ & $\begin{array}{l}\text { Eliminado } \\
\text { AFE }\end{array}$ \\
\hline 26 & $\begin{array}{l}\text { Me esforcé para conseguir } \\
\text { emprender mi negocio }\end{array}$ & Agencia & 4.46 & 0.86 & -1.71 & 2.70 & $.38^{*}$ & $.58^{*}$ & $\begin{array}{c}\mathrm{t}(113.35)=-11.52 \\
p<.001\end{array}$ & Agencia \\
\hline 27 & $\begin{array}{l}\text { Pensé en cómo podría hacerle para } \\
\text { emprender mi negocio }\end{array}$ & Rutas & 4.35 & 0.87 & -1.40 & 1.71 & $.32 *$ & $.50 *$ & $\begin{array}{c}\mathrm{t}(102.28)=-10.27 \\
p<.001\end{array}$ & $\begin{array}{l}\text { Eliminado } \\
\text { AFE }\end{array}$ \\
\hline 28 & Mantuve una actitud positiva & Agencia & 4.40 & 0.87 & -1.35 & 1.14 & $.37 *$ & $.59^{*}$ & $\begin{array}{c}\mathrm{t}(94.18)=-13.84 \\
p<.001\end{array}$ & $\begin{array}{l}\text { Eliminado } \\
\text { AFE }\end{array}$ \\
\hline 29 & $\begin{array}{l}\text { Pensé en las posibles } \\
\text { consecuencias de las decisiones } \\
\text { que tomaba }\end{array}$ & Rutas & 4.14 & 1.11 & -1.32 & 1.00 & $.28^{*}$ & $.54 *$ & $\begin{array}{c}\mathrm{t}(96.37)=-11.03 \\
p<.001\end{array}$ & $\begin{array}{l}\text { Eliminado } \\
\text { AFE }\end{array}$ \\
\hline
\end{tabular}

Nota. ${ }^{*} p<.001 ;{ }^{+}$Correlación por rangos de Spearman, reactivo-escala total. AFE $=$Análisis factorial exploratorio. 
Tabla 4. Matriz de configuración del instrumento según análisis factorial exploratorio

\begin{tabular}{|c|c|c|c|}
\hline Reactivos & $\begin{array}{c}\text { Agencia } \\
\omega=.88\end{array}$ & $\begin{array}{c}\text { Evaluación } \\
\text { de rutas } \\
\omega=.79 \\
\end{array}$ & $\begin{array}{c}\text { Planeación } \\
\text { alterna } \\
\omega=.64\end{array}$ \\
\hline Tuve interés en emprender mi negocio (23) & .86 & -.13 & -.08 \\
\hline Me comprometí para cumplir la meta de emprender mi negocio (24) & .78 & .02 & -.04 \\
\hline Tenía la firme idea de establecer mi negocio (22) & .70 & .05 & -.04 \\
\hline Me esforcé para conseguir emprender mi negocio (26) & .65 & .12 & -.09 \\
\hline Fui persistente para lograr mi objetivo de emprender mi negocio (13) & .63 & .07 & .06 \\
\hline Creí en mí para llegar a la meta de emprender mi negocio (6) & .63 & .07 & .03 \\
\hline Me creí capaz de emprender mi negocio (17) & .59 & .02 & .24 \\
\hline Creí en mi capacidad emprendedora (15) & .54 & -.05 & .23 \\
\hline Consideré los obstáculos a los que me enfrentaría (10) & .02 & .87 & -.12 \\
\hline Analicé que tan posible era lograr lo que tenía en mente (11) & .07 & .64 & .16 \\
\hline Tomé en cuenta los imprevistos que pudieran surgir (20) & .04 & .63 & .11 \\
\hline Tomé varios caminos para conseguir lo que tenía en mente (16) & .00 & -.04 & .63 \\
\hline Dividí en pequeños pasos el emprendimiento de mi negocio para lograrlo (14) & .11 & .03 & .62 \\
\hline Cambié mi planeación para lograr el emprendimiento, cuando fue necesario (12) & -.04 & .18 & .44 \\
\hline
\end{tabular}

Nota. Las cargas factoriales >.40 están en negritas. Método de extracción: Mínimos cuadrados no ponderados. ${ }^{+}$Rotación oblicua con normalización Kaiser, obtenida en 5 iteraciones. Entre paréntesis aparece el número de reactivo.

con cocientes de asimetría comprendidos entre 1.90 y -.66. Al encontrarse dentro de los límites recomendados, no se eliminó ninguno por este motivo. Asimismo, todas las opciones de respuesta posibles estuvieron representadas en cada reactivo.

En cuanto al análisis de grupos contrastantes, todos tuvieron un adecuado poder discriminativo (Ver Tabla 3).

\section{Validez de constructo: Análisis factorial exploratorio}

Se llevó a cabo un primer AFE con el método de extracción mínimos cuadrados no ponderados y con rotación oblimin directo. Los resultados de las pruebas Kaiser-Meyer-Olkin y Esfericidad de Bartlett, así como la determinante de la matriz de correlaciones mostraron que las correlaciones parciales y la covarianza común de los reactivos eran adecuadas para el análisis factorial ( $K M O=0.89$; prueba de esfericidad de Bartlett $\chi^{2}=1802.67, g l=91, p<.001$; determinante $>0$ ).

El primer análisis mostró una correlación entre factores de .13 a $.55 \mathrm{y}$, al tener más del $50 \%$ de correlaciones superiores a .32, se conservó el método de rotación oblimin directo. Se efectuó el AFE cinco veces en total, hasta obtener la estructura de tres factores final (ver Tabla 4), que explicó una varianza común de $49.36 \%$. Para ello, se eliminaron 12 reactivos $(1,3,4,5,7,8,9,18$, $19,21,28,29)$ por no cargar en ningún factor y 3 reactivos $(2,25,27)$ por no tener congruencia conceptual en el factor en el que se ubicaron. Es importante mencionar que el reactivo 12 tuvo una comunalidad de .27 , pero se dejó por tres razones:
(1) su carga factorial era adecuada, (2) mantenía la estructura conceptual y (3) no mejoraba la confiabilidad del instrumento si se eliminaba. Las tres dimensiones obtenidas proporcionaban una estructura factorial clara e interpretable de acuerdo con el enfoque teórico de Snyder (1995) y fueron denominadas: Agencia, Evaluación de rutas y Planeación.

\section{Coeficientes de confiabilidad}

Las tres dimensiones obtenidas tuvieron los siguientes valores de confiabilidad : Agencia (8 reactivos, $\omega=.88)$, Evaluación de rutas (3 reactivos, $\omega=.79)$ y Planeación alterna (3 reactivos, $\omega=.64)$.

\section{Diferencias entre mujeres emprendedoras y multinivel: Validez predictiva}

Ni la escala ni los factores tuvieron una distribución normal, por ello se utilizó la prueba U de Mann-Whitney para determinar las diferencias entre los grupos de la muestra. Tal como se esperaría de un instrumento diseñado para medir esperanza para emprender, se encontraron diferencias estadísticamente significativas en las puntuaciones de la escala total y de Agencia y Evaluación de Rutas (ver Tabla 5). Todo lo cual quiere decir que esta escala es capaz de encontrar las diferencias esperadas entre las mujeres emprendedoras y las multinivel. 
Tabla 5. Diferencias en las puntuaciones de la EEEM entre mujeres emprendedoras y multinivel

\begin{tabular}{|c|c|c|c|c|c|c|c|c|}
\hline \multirow[b]{2}{*}{ Dimensiones } & \multicolumn{3}{|c|}{$\begin{array}{l}\text { Emprendedoras } \\
\quad \mathrm{n}=165\end{array}$} & \multicolumn{3}{|c|}{$\begin{array}{c}\text { Multinivel } \\
\mathrm{n}=166\end{array}$} & \multirow[b]{2}{*}{$\begin{array}{c}\text { U } \\
\text { Mann- } \\
\text { Whitney }\end{array}$} & \multirow[b]{2}{*}{$p$} \\
\hline & $\begin{array}{l}\text { Rango de } \\
\text { medias }\end{array}$ & $\begin{array}{l}\text { Suma de } \\
\text { rangos }\end{array}$ & $M d n$ & $\begin{array}{l}\text { Rango de } \\
\text { medias }\end{array}$ & $\begin{array}{l}\text { Suma de } \\
\text { rangos }\end{array}$ & $M d n$ & & \\
\hline Esperanza para emprender & 179.51 & 29619 & 61 & 152.57 & 25327 & 58 & 11466 & .01 \\
\hline Agencia & 181.82 & 30000 & 38 & 150.28 & 24946 & 36 & 11085 & $<.01$ \\
\hline Evaluación de rutas & 179.60 & 29634 & 12 & 152.48 & 25312 & 11 & 11452 & $<.01$ \\
\hline Planeación alterna & 171.22 & 28251 & 11 & 160.81 & 26695 & 11 & 12834 & .32 \\
\hline
\end{tabular}

\section{Discusión}

Los factores encontrados fueron acordes con la teoría de Snyder (1995), la cual originalmente contenía dos dimensiones: agencia y rutas. En torno a este modelo de dos factores se teoriza y se construyen instrumentos de corte cognoscitivo (véase Babyak et al., 1993; Snyder, Harris et al., 1991; Snyder et al., 1997).

Derivado del primer jueceo de reactivos, tuvimos que clarificar el constructo y sus dimensiones, pues había traslape entre ellas. Pese a notar que, en las rutas de acción para el logro se incluyeron procesos cognoscitivos de dos tipos: (1) los destinados a evaluar la meta y (2) a desarrollar varias formas de alcanzarla (Hutz et al., 2014; Snyder, Irving et al., 1991; Snyder et al., 1996), se dejó como una sola dimensión, como indicaba la teoría. Esta división conceptual que observamos en las rutas de acción de Snyder (1995) se corroboró psicométricamente en el modelo de tres factores aquí presentado, al hallar las dimensiones: Evaluación de rutas y Planeación alterna.

La evaluación de rutas se definió como el análisis de las circunstancias en las que ocurrirá el logro, para determinar si la meta puede conseguirse e identificar los posibles obstáculos para incluirlos en la planeación. La planeación alterna es la división de las acciones destinadas al logro en pasos más pequeños, que especifican la secuencia de acciones para su alcance y adecuarlas, de ser necesario.

Snyder et al. (1997) indican que basta utilizar el puntaje total de la escala para determinar la validez convergente, discriminante y predictiva del constructo. Por ende, las diferencias encontradas entre mujeres emprendedoras y multinivel multinivel proporcionan indicios adecuados de la validez predictiva de la escala. Esto es congruente con los desafíos y riesgos que enfrenta una emprendedora, quien requiere mayor cantidad de recursos personales, financieros y de infraestructura, por lo que su esperanza para emprender debe ser superior. Por ende, los valores de validez fueron adecuados, tanto psicométrica, como conceptualmente.

Los coeficientes de confiabilidad, según el omega de McDonald, fueron adecuados $\mathrm{y}$ parecidos a los encontrados en otras escalas (ver Tabla 1). Snyder, Harris et al. (1991) hallaron coeficientes de confiabilidad (alpha de Cronbach) que van de .71 a .76 para agencia y de .63 a .68 para rutas; esta última alcanza .80 en una muestra de ocho exploradas. La confiabilidad baja de Planeación alterna coincidió con los valores que tuvo rutas en la investigación antes citada, sin embargo, el de Evaluación de rutas fue superior. Lo primero podría deberse a que incluye procesos mentales relacionados con la premeditación y la planeación, además de la flexibilidad, como consta en el reactivo 12. Por consiguiente, se trata de una dimensión compleja que, quizá requiera mayor cantidad de reactivos.

Ya que la escala EEEM posee valores adecuados de confiabilidad y validez de contenido y de constructo, se podría utilizar como una herramienta de evaluación, diagnóstico e investigación en la esperanza y el emprendimiento $\mathrm{y}$, adicionalmente, en otros contextos en los cuales se estudie el logro de metas. Una de sus ventajas es la claridad conceptual de las dimensiones y de sus reactivos. En la escala derivada del modelo teórico empleado (Snyder, Harris et al., 1991), los reactivos son más generales, como una disposición general basada en el pasado y el presente, con cuatro reactivos por dimensión (i.e. 
Agencia: He sido muy exitoso en la vida; Rutas: Puedo pensar en muchas maneras de salir de un atolladero). Los reactivos de agencia refieren únicamente a las metas conseguidas o a los resultados previos, pero no a la capacidad de la persona para lograrlas, mientras que los de rutas no contemplan los posibles obstáculos, como remite la definición original.

Una de las limitantes del estudio fue la representatividad de la muestra. Esto podría superarse en futuros estudios que incluya diferentes regiones que representen la riqueza sociocultural del país.

Es indispensable comprobar que las propiedades psicométricas de la estructura del EEEM, que aquí se presenta, se mantienen en otra muestra, mediante un AFE o un Análisis factorial confirmatorio (AFC), ya que la eliminación de algunos reactivos puede afectar la respuesta de los que se conservan. Mientras tanto, a quien requiera aplicar el instrumento, se le sugiere incluir los reactivos originales, en el orden señalado en la tabla 3, aunque únicamente se hagan los análisis con los 14 que quedaron en el AFE expuesto en este artículo.

En versiones posteriores de la escala podría explorarse la división del factor Agencia, ya que trata sobre dos aspectos claramente diferenciados: (1) la creencia sobre la certeza de emprender un negocio (Dixson et al., 2017) que es propiamente la agencia, y (2) la motivación para conseguir las metas, es decir, motivación. Como se dijo anteriormente, la escala original sólo contempla lo relacionado con logro de metas como enunciados generales (véase Snyder, Harris et al., 1991). Es necesario aclarar que las definiciones elaboradas para este estudio, contemplan las publicaciones de C. R. Snyder posteriores a la generación de su escala de esperanza.

En conclusión, la EEEM es una herramienta útil para medir la esperanza desde un corte cognoscitivo, vinculada con el logro de metas y con el emprendimiento, ya que hasta el momento no se ha encontrado otra similar. Es asimismo un buen avance para el estudio de este constructo, ya que ahora se cuenta con un instrumento válido y confiable, con adecuado soporte psicométrico.

\section{Referencias}

Alvaro Fariñas, A. (2015). Qué es una empresa multinivel y cómo funciona. Recuperado de https://noticias.infocif.es/noticia/que-es-unaempresa-multinivel-y-como-funciona

Babyak, M. A., Snyder, C. R., \& Yoshinobu, L. (1993). Psychometric properties of the hope scale: A confirmatory factor analysis. Journal of Research in Personality, 27(2), 154-169. doi:10.1006/jrpe.1993.1011

Bai, K., Kohli, S., \& Malik, A. (2017). Self efficacy and hope as predictors of mental health. Indian Journal of Positive Psychology, 8(4), 631-635.

Beavers, G. A., Iwata, B. A., \& Lerman, D. C. (2013). Thirty years of research on the functional analysis of problem behavior. Journal of applied Behavior Analysis, 46(1), 1-21. doi:10.1002/jaba.30

Bruininks, P., \& Malle, B. F. (2005). Distinguishing hope from optimism and related affective states. Motivation and Emotion, 29(4), 327-355. doi:10.1007/s11031-006-9010-4

Bullough, A., \& Renko, M. (2013). Entrepreneurial resilience during challenging times. Business Horizons 56, 343-350. doi:10.1016/j.bushor.2013.01.001

Callina, K. S., Snow, N., \& Murray, E. D. (2018). The history of philosophical and psychological perspectives on hope: Toward defining hope for the science of positive human development. En M. W. Gallagher \& S. J. Lopez (Eds.). The Oxford handbook of hope (pp. 5-9). Reino Unido: Oxford. doi: 10.1093/oxfordhb/9780199399314.001.0001

Castiblanco, S. E. (2013). La construcción de la categoría de emprendimiento femenino. Revista de la Facultad de Ciencias Económicas: Investigación y Reflexión, 21(2), 53-66.

Castiblanco, S. E. (2016). Female entrepreneurship in a forced displacement situation: The case of Usme in Bogota. Suma de Negocios, 7, 61-72. doi:10.1016/j.sumneg.2016.02.004

Clark-Carter, D. (2004). Quantitative psychological research: A student's handbook. EE.UU.: Psychology Press. 
Covin, J. G., \& Wales, J. G. (2011). The measurement of entrepreneurial orientation. Entrepreneurship theory and practice, 36(4), 677-702. doi:10.1111/j.1540-6520.2010.00432.x

Diccionario de etimologías de Chile (2019). Recuperado de http://etimologias.dechile.net/?esperanza

Dixson, D. D., Worrell, F. C., \& Mello, Z. (2017). Profiles of hope: How clusters of hope relate to school variables. Learning and Individual Differences, 59, 55-64. doi:10.1016/j.lindif.2017.08.011

Erickson, R. C., Post, R. D., \& Paige, A. B. (1975). Hope as a psychiatric variable. Journal of Clinical Psychology, 31, 324-330. doi:10.1002/1097-4679(197504)31:2<324::AIDJCLP2270310236>3.0.CO;2-Q

Etchegoyen, J. F. (2019). Financiar a las emprendedoras mexicanas es un buen negocio. Forbes México.

https://www.forbes.com.mx/financiar-a-lasemprendedoras-mexicanas-es-un-buennegocio/

Ferrando, P. J., \& Anguiano-Carrasco, C. (2010). El análisis factorial como técnica de investigación en psicología. Papeles del Psicólogo, 31(1), 18-35.

Flora D. B., LaBrish, C., \& Chalmers, R. P. (2012). Old and new ideas for data screening and assumption testing for exploratory and confirmatory factor analysis. Frontiers in Psychology (Quantitative Psychology and Measurement), 3(55), 1-2. doi:10.3389/fpsyg.2012.00055

Gallagher, M. W. (2018). Introduction to the science of hope. En M. W. Gallagher \& S. J. Lopez (Eds.), The Oxford Handbook of Hope (pp. 5-9). doi:10.1093/oxfordhb/9780199399314.001.0001

Global Entrepreneurship Monitor (GEM). (2017). Global report 2016/17. Recuperado de https://www.gemconsortium.org/report/gem2016-2017-global-report

Gottschalk, L. (1974). A hope scale applicable to verbal samples. Archives of General Psychiatry, 30, 779-785.

doi:10.1001/archpsyc.1974.01760120041007
Hair, J. F., Bush, R. P., \& Ortinau, D. J. (2010). Investigación de mercados: En un ambiente de información digital. México: McGrawHill.

Hert, K. (1991). Development and refinement of an instrument of hope. Journal of advanced nursing, 17, 1251-1259.

Hutz, C., Midgett, A., \& Cerentini Pacico, J. (2014). The relationship of hope, optimism, self-esteem, subjective well-being, and personality in Brazilians and Americans. Psychology, 5, 514-522. doi:10.4236/psych.2014.56061

Jaramillo, L. (2008). Emprendimiento: Concepto básico en competencias. Lumen-Instituto de Estudios en Educación, 7, 1-6.

Krueger, N. F., Reilly, M. D., \& Carsrud, A. L. (2000). Competing models of entrepreneurial intentions. Journal of Business Venturing, 15(5), 411-432.

Lambing, P., \& Kuehl, C. (1998). Empresarios pequeños y medianos. México: Prentice Hall.

Liñán, F., \& Chen, Y. W. (2009). Development and cross-cultural application of a specific instrument to measure entrepreneurial intentions. Entrepreneurship Theory and Practice, 33(3), 593-617. doi:10.1111/j.1540-6520.2009.00318.x

Lopez, S. J., Snyder, C. R., \& Teramoto, J. (2004). Hope: Many definitions, many measures. En S. J. Lopez \& C. R. Snyder (Eds.), Positive psychological assessment: A handbook of models and measures (pp. 91106). EE.UU.: American Psychological Association. doi:10.1037/10612-000

Luthans, F., \& Youssef, C. (2004). Human, social, and now positive psychological capital management: Investing in people for competitive advantage. Organizational Dynamics, 33(2), 143-160.

Mahfud, T., Triyono, M. B., Sudira, P., \& Mulyani, Y. (2019). The influence of social capital and entrepreneurial intentions: The mediating role of psychological capital. European Research on Management and Business Economics, 26, 33-39.

Markussen, S., \& Roed, K. (2017). The gender gap in entrepreneurship. The role of peer effects. Journal of Economic Behavior \& Organization, 134, 356-373. doi:10.1016/j.jebo.2016.12.013 
Martínez Uribe, P., Cassaretto Bardales M., \& Hert, K. (2012). Propiedades psicométricas de la escala de esperanza de Herth en español. Revista Iberoamericana de Diagnóstico y Evaluación - e Avaliação Psicológica, 33(1), 127-145.

McDonald, R. P. (1999). Test theory: A unified treatment. Mahwah: Lawrence Erlbaum Associates, Inc.

McGuire-Snieckus, R. (2014). Hope, optimism and delusion. Psychiatric Bulletin, 38, 49-51. doi:10.1192/pb.bp.113.044438

McMullen, J., \& Shepherd, D. A. (2006). Entrepreneurial action and the role of uncertainty in the theory of the entrepreneur. Academy of Management Review, 31, 132142.

Miller, J. F., \& Powers, M. J. (1988). Development of an instrument to measure hope. Nursing Research, 37(1), 6-10. doi:10.1097/00006199-198801000-00002

Montero, I., \& León, O. G. (2007). A guide for naming research studies in Psychology. International Journal of Clinical and Health Psychology, 7, 847-862.

Moriano, J. A., Gorgievski, M. J., Laguna, M., \& Stephan, U. (2012). A cross-cultural approach to understanding entrepreneurial intention. Journal of Career Development, 39(2), 162185. doi:10.1177/0894845310384481

Moses, C. L., Olokundun, M. A., Akinbode, M., Agboola, M., \& Inelo, F. (2016). Entrepreneurship education and entrepreneurial intentions: The moderating role of passion. The Social Sciences, 11, 645653.

Nowotny, M. L. (1989). Assessment of hope in patients with cancer: Development of an instrument. Oncology nurse forum, 16(1), 5761.

Nunnally, J. C. (1991). Teoría psicométrica. México: Trillas.

Onuoha, G. (2007). Entrepreneurship. AIST International Journal, 10, 20-32.

Obayuwana, A. O, Collins, J. L., Carter, A. L., Rao, M. S., Mathura, C. C., \& Wilson, S. B. (1982). Hope index scale: An instrument for the objective assessment of hope. Journal of the National Medical Association, 74, 761765.
Oettingen, G. (1997). Psychologie des zukunftsdenkens: The psychology of thinking about the future. Germany: Hogrefe.

Oettingen, G., \& Gollwitzer, P. M. (2002). Turning hope thoughts into goal-directed behavior. Psyhological Inquiry, 13, 304-307.

Oliveira, E. P., Merino, M. D., Privado, J., \& Almeida, L. S. (2017). Escala de funcionamento psicológico positivo: Adaptação e estudos iniciais de validação em universitários portugueses. Revista Iberoamericana de Diagnóstico y Evaluación - e Avaliação Psicológica, 3(48), 151-162. doi:10.21865/RIDEP48.3.13

Osowska, R., Kapasi, I., \& Jackman, L. (Junio, 2016). The effect of culture on female entrepreneurship motivations. Fifth international workshop: Entrepreneurship. En Culture, finance and economic development, Lyon, Francia.

Paes, F. J. (2002). O Retrato Falado de una Megatendência (Tesis de Licenciatura). Universidade Católica de Goiás, Goiás, Brasil.

Portillo Navarro, M. J., \& Millán Jiménez, A. (2016). Moderators elements of entrepreneurship: Gender differences. Suma de negocios, 7, 47-53.

doi:10.1016/j.sumneg.2016.02.002

Ramos, V., Herrera, L., Franco-Crespo, A., Guerra, Y., González-Pérez, L., RamosGalarza, C. ... (2020). Validación del cuestionario OLC (organizational learning culture) en organizaciones ecuatorianas. Revista Iberoamericana de Diagnóstico y Evaluación - e Avaliação Psicológica, 56(3), 159-169.

Rubio, A., \& Esteban, N. (2016). Cultural factors and gender role in female entrepreneurship. Suma de negocios, 7, 9-17. doi:10.1016/j.sumneg.2015.12.002

Scioli, A., Chamberlin, C. M., Samor, C. M., Lapointe, A. B., Campbell, T. L., \& Macleod, A. R. (1997). A prospective study of hope, optimism, and health. Psychological Reports, 81, 723-733. doi:10.2466/pr0.1997.81.3.723

Snyder, C. R. (1995). Conceptualizing measuring and nurturing hope. Journal of Counseling and Development, 73(3), 355-360.

Snyder, C. R., Harris, C., Anderson, J. R., Holleran, S. A., Irving, L. I., Sigmon, S. X. ... 
(1991). The will and the ways: Development and validation of an individual-differences measure of hope. Journal of Personality and Social Psychology, 60, 570-585.

Snyder, C. R., Hoza, B., Pelham, W. E., Rapoff, M., Ware, L., Danovsky, M. ... (1997). The development and validation of the children's hope scale. Journal of Pediatric Psychology, 22, 399-421.

Snyder, C. R., Irving, L., \& Anderson, J. R. (1991). Hope and health: Measuring the will and the ways. En C. R. Snyder \& D. R. Forsyth (Eds.), Handbook of social and clinical psychology: The health perspective (pp. 285-305). EE.UU.: Pergamon Press.

Snyder, C. R., Lopez, S. J., Shorey, H. S., Rand, K. L., \& Feldman, D. B. (2003). Hope theory, measurements, and applications to school psychology. School Psychology Quarterly, 2(18), 122-139.

Snyder, C. R., Rand, K. L., \& Sigmon, D. R. (2002). Hope theory: A member of the positive psychology family. En C. R. Snyder \& S. J. Lopez (Eds.), Handbook of positive psychology (pp. 257-276). EE.UU.: Oxford University Press.

Snyder, C. R., Sympson, S. C., Ybasco, F. C., Borders, T. F., Babyac, M. A., \& Higgins, R. L. (1996). Development and validation of the state hope scale. Journal of Personality and Social Psychology, 70(2), 321-335. doi:10.1037//0022-3514.70.2.321

Stoner, M. H., \& Keampfer, S. H. (1985). Recalled life expectancy information, phase of illness and hope in cancer patients. Research in Nursing and Health, 8, 269-274.

Stotland, E. (1969). The psychology of hope. San Francisco, CA: Jossey-Bass.

Tabachnick, B., \& Fidelli, L. (2001). Using multivariate statistics. EE.UU.: Harper \& Row.

Valle, M. F., Huebner, E. S., \& Suldo, S. M. (2006). An analysis of hope as a psychological strength. Journal of School Psychology, 44, 393-406. doi:10.1016/j.jsp.2006.03.005

Vargas Núñez, I. (1995). Construcción y validación de una escala que evalúe la esperanza en México (Tesis de Maestría).
Universidad Nacional Autónoma de México, Ciudad de México, México.

Villacieros, M., Bermejo, J. C., \& Hassoun, H. (2017). Validación de la escala de esperanza en enfermedad terminal para familiares breve (EEET-fb): Análisis de validez y fiabilidad. Anales del sistema sanitario de Navarra, 40(3), 391-400. doi:10.23938/ASSN.0118

Williams, B. A., Onsman, A., \& Brown, G. T. (2010). Exploratory factor analysis: A fivestep guide for novices. Journal of Emergency Primary Health Care, 8(3), 1-13.

Ximénez, M. C., \& García, A. G. (2005) Comparación de los métodos de estimación de máxima verosimilitud y mínimos cuadrados no ponderados en el análisis factorial confirmatorio. Psichotema, 17(3), 528-535.

Yang, Y., Zhang, M., \& Kou, Y. (2016). Selfcompassion and life satisfaction: The mediating role of hope. Personality and Individual Differences, 98, 91-95. doi:10.1016/j.paid.2016.03.086 